\title{
OBESITY STIGMA: THE ROLE OF HEALTHCARE PRACTITIONERS IN IMPROVING PATIENT OUTCOMES
}

\author{
Olivia Austen
}

\section{INTRODUCTION}

Obesity stigma is a common occurrence for people that are labelled either by society or more often by the measure of body mass index (BMI) as 'obese' or 'overweight.' Within healthcare, perpetuation of this stigma occurs at alarming rates with harmful psychological and physiological repercussions for patients (Phelan et al., 20I5). It is the professional responsibility of healthcare practitioners to ensure that the care they provide is nonstigmatising and ethical for all patients, but commonly this is not the case. Isolation of people from healthcare and other personal support networks can be a response to experiencing stigmatising healthcare. (Puhl \& Brownell, 2006). Isolation due to obesity stigma can be linked to this year's SCOPE Health \& Wellbeing theme of islands, as patients being isolated from adequate healthcare is similar to an island being disconnected from the mainland. In this analogy, stigma experienced is the boundary that separates those experiencing it from healthcare and further can cause avoidance of healthcare (Phelan et al., 2015). Isolation from healthcare services needs to be avoided if therapeutic relationships between healthcare professionals and patients are to be preserved. This article will explore the effects of obesity stigma on patients, the source of these attitudes and the role of healthcare professionals in improving care for all higher-weight patients, and will make two suggestions for how the isolating impact of stigmatisation can be remedied. This article has been written from a nursing perspective but the findings and information included are applicable to all healthcare professions and their interactions with higher-weight patients.

\section{BACKGROUND}

In order to understand the issue of obesity stigma, first an understanding of the terms used in this article is required. The use of terms 'overweight' and 'obese' are recognised as contributing to weight-based stigma, but will be used in this article to form a connection to the terminology used within the reviewed evidence-based literature. The terms 'overweight' and 'obese' are used in relation to their categories under the body mass index. The terms weight stigma, weight bias, obesity bias and obesity stigma are used interchangeably throughout this article. In New Zealand and around the world, being overweight is classified as having a BMl of $25 \mathrm{~kg} / \mathrm{m} 2$ or more, and being obese as a BMl of $30 \mathrm{~kg} / \mathrm{m} 2$ or more (Ministry of Health [MOH], 20l7; World Health Organization [WHO], 2020). $\mathrm{BMI}$ is the international standardised measure for indicating and diagnosing overweight and obesity in patients, but some research has found that it is not always an accurate indicator of increased risk for morbidity and mortality, and consequently obesity does not always signify an increase in adverse health risks (Essayli, Murakami, Wilson \& Latner, 2017; Tylka et al., 2014). People who are outside of 'average weight' BMI range can still be 'healthy' and equally people with an 'average' BMI can still be 'unhealthy' (Bacon \& Aphramor, 20II; Tylka et al., 20I4). Research by Puhl and Brownell (2006) indicates that people who are categorised as overweight or obese by $\mathrm{BMI}$, experience stigma in over half of their interactions with healthcare professionals (69 per cent from doctors and 46 per cent from nurses). Patients' experiences of weight stigma from healthcare professionals can include, insufficient non-verbal communication (lack of eye contact), inappropriate comments and use of undesirable 
weight descriptors (that is, 'too fat' and the terms 'overweight' and 'obesity'), rejection, and negative assumptions about abilities (Essayli et al., 2017; Puhl \& Brownell, 2006; Wakefield \& Feo, 2017). These incidents happen due to health professionals learned and internalised weight bias. The stigma surrounding people who are overweight or obese often includes an assumption that they are 'unhealthy' for the reason that they do not fit into the 'averageweight' BMI category (I8.5-24.9 kg/m2), and stereotypical assumptions that they are weak-willed, stupid, lack selfdiscipline and personal control (Phelan et al., 2015; Puhl \& Brownell, 2006; Tylka et al., 2014). Stereotypes about people with obesity impact the care provided by healthcare professionals (Phelan et al., 2015). In New Zealand, 66.2 per cent of the population is considered overweight or obese $(\mathrm{MOH}, 2020 \mathrm{a})$, with about one in three adults (30.9 per cent) falling into the obese category $(\mathrm{MOH}, 2020 \mathrm{~b})$. These statistics represent a large portion of the population that have the potential to be negatively impacted by weight bias in Aotearoa.

\section{DISCUSSION}

\section{Sources of Weight-Bias Attitudes}

Origins behind health professionals' strong negative attitudes and preconceptions about weight often come from the education they receive during training. The main focus on obesity in this training, is that it should be seen an avoidable risk factor, and this misconstrues weight as something that can be controlled solely through determination and appropriate diet and exercise (Phelan et al., 2015; Puhl \& Brownell, 2006; Tylka et al., 20l4). This places unfair blame on overweight and obese people regarding their weight. Obesity is widely viewed by those in the field of healthcare as being a risk factor for illness and disease, however data suggests that this is not the case. The evidence presented in Bacon and Aphramor (20II) indicates that a higher BMI is linked to, but not the cause of, increased risk for disease, and also states that due to the 'obesity paradox' higher BMI has been associated with decreased mortality when compared to people with lower BMI, in certain diseases (chronic kidney disease, hypertension and so on) (Bacon \& Aphramor, 20II; Puhl \& Brownell, 2006). It is also important to highlight here that the medical model of health often uses a simplistic view of obesity, one that does not take into account that there are many more factors to obesity than just diet and exercise; genetics, environment, social and other factors also influence weight (Phelan et al., 2015). The attitudes that healthcare professionals hold about people with overweight and obesity can impact the way they provide care to these patients, this can happen through preconceptions being translated into discriminatory behaviours (Puhl \& Brownell, 2006). This may occur through implicit and explicit means. Examples of behaviours that implicit and explicit attitudes may result in are, respectively, poor non-verbal communication and alterations in decision making (Phelan et al., 2015).

\section{Effects of Stigmatising Healthcare Experiences}

Common themes that appeared throughout the literature include wide-ranging impacts on physiological health and psychological well-being (Phelan et al., 2015). Physical effects include exposure to high levels of stress hormones such as cortisol (which over time can have multiple negative health impacts) and not being taken seriously by health professionals for other non-weight related health concerns, resulting in lack of adequate treatment and the prolongation of health issues (bias causes health professionals to assume the health concern being presented is related to excess weight) (Phelan et al., 2015). Psychological effects from being subjected to weight bias attitudes from health professionals can include risk of depression, negative self-talk, lower self-esteem and disordered eating (Essayli et al., 20I7; Phelan et al., 2015; Puhl \& Brownell, 2006; Puhl, Himmelstein, Gorin, \& Suh, 2017).

Experiencing weight stigma in a range of healthcare settings has also been shown in correlation with patient disengagement with health services, decrease in perceived quality of care, less patient-centred care and a mistrust of health professionals by patients with overweight or obesity (Phelan et al., 20l5). Thus it can be inferred that weight stigma can have a major negative impact on the likeliness of an overweight or obese patient accessing essential healthcare services. 


\section{Weight-Normative vs. Weight-Inclusive Approaches to Care}

Patient-centred, non-stigmatising healthcare on the other hand, can help reduce the patient perceived threat of conversations about weight (Phelan et al., 2015). A case study by Wakefield and Feo (2017) showed that common impacts of non-stigmatising care can include an increase in positive feelings and self-esteem and increased ability to self-manage. Non-stigmatising, person-centred care also promoted spiritual and emotional well-being, and resulted in improved patient outcomes (Wakefield \& Feo, 2017).

A review of weight-normative and weight-inclusive approaches to health by Tylka et al., (2014) found that the universally used, weight-normative approach (which emphasises weight as the main determinant of health and where the key focus is on weight management and loss to treat and prevent a variety of health problems and generally improve health) is not effective for the majority of people, as they are likely to regain previously lost weight, and continue the process of weight cycling (losing and gaining weight periodically). With a weight-normative approach, patients are more likely to experience and internalise weight stigma, and are therefore more likely to suffer its harmful effects of body shame, body dissatisfaction, and disordered eating (Tylka et al., 2014).

The inverse of this is the weight-inclusive approach. This approach to health focuses on working holistically with patients and shifts the attention from weight loss to being and achieving health, independent of weight (Tylka et al., 2014). This is accomplished by bringing attention to the complexity of obesity, and an appreciation that there is no 'normal' size that bodies should be, it recognises that variety in body size and shape is natural and that there is no one size that a person needs to be in order to achieve health. It also refutes the well-established idea with the healthcare community that a higher BMI is a direct cause of poor health (Tylka et al., 2014). Weight-inclusive care is non-stigmatising and patient-centred, and is about assessing the individual needs of individual patients. This approach reduces weight stigma experienced by patients in the healthcare setting.

One of the models of weight-inclusive healthcare that the review by Tylka et al. (2014) supports is the Health at Every Size (HAES) model. This model's purpose is to shift the driving factor of health away from weight loss towards an improvement in health promoting behaviours (Bacon \& Aphramor, 20II). This is done through recommending and education around intuitive eating and encouraging body acceptance (Bacon \& Aphramor, 20II; Tylka et al., 2014). Intuitive eating is where instead of using a prescribed diet or restricting any food or food groups, patients are encouraged to notice and become aware of their body's signals, to eat when they actually feel hungry, and to cease eating when they feel full (Bacon \& Aphramor, 20II). This type of regulation of food consumption is related to improved intake of nutrients, and reduced disordered eating. Although there have been concerns that a body positive and accepting approach to obesity would cause an increase in the amount of people with obesity, this is not supported by evidence (Bacon \& Aphramor, 20II).

A weight-inclusive and non-stigmatising approach to health upholds the bioethical principles of beneficence and nonmaleficence and has implications for all healthcare practitioners to keep the focus on being patient-centred and non-judgemental (Bacon \& Aphramor, 20II; Tylka et al., 2014). The relevance of this evidence-based literature to nursing practice is that within the healthcare workforce, nurses make up the majority, and thus it falls to the profession of nursing to become leaders in championing the drive to eradicate weight stigma from all healthcare practice (Wakefield \& Feo, 2017). The benefits of this evidence is that there is already a patient-centred basis for change, and there are already established and tested ideas and approaches which can be used to help inform future strategies to overcome healthcare professional weight stigma, and to help patients cope with experienced weight bias (Phelan et al., 2015; Puhl \& Brownell, 2006). Two recommendations follow on how these goals, however ambitious they may seem, could lead to increased quality of care and health outcomes for higher-weight people. 


\section{RECOMMENDATIONS}

\section{Education Programs for Health Professionals}

One evidence-based recommendation that would be likely to decrease obesity stigma in the context of healthcare, would be to have focused education programs for health professionals regarding the complexity of obesity and obesity care (Phelan et al., 2015). This would be most beneficial if integrated throughout all healthcare professions' curricula, so that students and current practitioners would have the same levels of knowledge on this complex issue. This education would need to concentrate on the multifaceted causes for obesity and the need for nonstigmatising care for people with overweight and obesity, and would include identifying the ways that health professionals carry obesity bias and how this can translate to discrimination of patients. This education would also need to highlight the negative health impacts of obesity stigma for patients.

Education programs could include the use of weight-neutral, non-stigmatising language when discussing weight with patients in order to keep the patient engaged and reduce their negative views of the healthcare system. The rationale behind implementing this sort of educational training for nurses and other health professionals in New Zealand lies with the positive impacts on patients, which include increases in positive feelings and selfesteem, increases in spiritual and emotional well-being, and improved patient outcomes (Bacon \& Aphramor, 20II). It ensures that the care provided by nurses is effective, compassionate, inclusive, and based on best practice evidence. Once the evidence-based facts are known, nurses and other healthcare professionals are able to gain the competence to implement change in their practice.

\section{Making the Shift to a Weight-Inclusive Model of Health}

The second recommendation to benefit patient care would be pushing for change from the current weightnormative to a weight-inclusive model of health. This should be within national health policy, but also driven by individual practitioners in the healthcare sector. The implementation of this type of model could be accomplished by nurse-led initiatives, such as education sessions for all healthcare professionals, around a weight-inclusive approach to care. The Health At Every Size (HAES) framework is an evidenced-based example of a weightinclusive model that can be used to promote body acceptance and long-term healthy behavioural changes (Bacon, Stern, Van Loan, \& Keim, 2005; Bacon \& Aphramor, 201I).

Specific changes to practice could include a push for patient inclusive motivational interviewing, and a focus on forming habits that promote health rather than focusing solely on weight loss. Intuitive eating and educating patients on how to tune into signals of hunger and satiety (as well as how their body and mood responds to different foods), allows for the formation of lasting habits rather than the short-lived results of restrictive dieting (Bacon \& Aphramor, 20II). Promoting self-acceptance is another key aspect of the HAES approach; compassionfocused behaviour change theory suggests that having higher self-esteem makes a person more likely to implement positive health changes (Bacon \& Aphramor, 20II). This can be achieved through helping patients to identify the value of their bodies as they are, despite how this may differ to a desired body weight or image (Bacon \& Aphramor, 20lI). Focusing on what a patient's body is able to do and the activities that they enjoy creates an environment of well-being and self-care rather than forcing them into a generalised box that says they must exercise for a set time however many times a week, in a certain manner. Personalising care and shifting the focus from weight loss to well-being is the essence of a weight-inclusive model of health.

Based on the previously presented evidence, the clinical outcomes of implementing this type of change would include an increase in patient self-esteem, body satisfaction and adherence to behavioural change recommendations, and would also seek to address systemic and institutionalised weight discrimination within healthcare (Tylka et al., 20I4). 


\section{CONCLUSION}

Due to the high rates of overweight and obesity in New Zealand, it is important the care that nursing and other health professionals provide is non-stigmatising and free of bias to improve the health and well-being of patients with overweight and obesity. In order to accomplish this, there must be adequate education of students and current practitioners alike to recognise and challenge internally-held weight biases. In order to provide more effective, beneficial, stigma-free care to higher-weight patients, there should be a shift in language used when talking about weight with patients. A global healthcare move towards a weight-inclusive system of health is a way of increasing patient benefits and moving towards inclusive, ethical, evidence-based care. This will also assist in minimising the isolating effects of obesity stigma for patients and allow them to reconnect with healthcare services. This reconnection can be likened to that of a boat that restores connection to an isolated island after a storm; through weight-inclusive healthcare those people isolated through obesity stigma can be brought back to the mainland of evidence-based healthcare. People whose bodies are obese or overweight deserve the same level of respect and care as any other patient and deserve to not be discriminated against within the realm of healthcare. To accomplish this, the preconceived attitudes of health practitioners about weight must be challenged to address this issue of obesity stigma, and to inform best patient care.

Olivia Austen is a third-year student completing her Bachelor of Nursing at Otago Polytechnic in Dunedin, New Zealand. She is interested in understanding how health practitioner biases can affect patient care and outcomes. She has a passion for ensuring nursing care is patient-centred, compassionate, and free of stigma.

Correspondence to: Olivia Austen. Email: olivia.austen13@gmail.com 


\section{REFERENCES}

Bacon, L., Stern, J., Van Loan, M., \& Keim, N. (2005). Size acceptance and intuitive eating improve health for obese, female chronic dieters. Journal of the American Dietetic Association, 105(6), 929-936. doi:10.1016/j.jada.2005.03.011.

Bacon, L., \& Aphramor, L. (20II). Weight science: Evaluating the evidence for a paradigm shift. BMC Nutrition Journal, 10(9). doi:|0.||86/|475-289|-10-9.

Essayli, J., Murakami, J., Wilson, R., \& Latner, J. (2017). The impact of weight labels on body image, internalized weight stigma, affect, perceived health, and intended weight loss behaviours in normal-weight and overweight college women. American Journal of Health Promotion, 31(6), 484-490. doi:10.1177/0890117116661982.

Ministry of Health. (2017). Clinical guidelines for weight management in New Zealand Adults. Wellington, New Zealand: Author.

Ministry of Health. (2020a). Annual Data Explorer: Overweight or obese: BMI of 25.0 or greater (or IOTF equivalent for 15-17 years). Retrieved from https://minhealthnz.shinyapps.io/nz-health-survey-2019-20-annual-data-explorerl_w_e809clb0/\#!/exploreindicators

Ministry of Health. (2020b). Obesity Statistics. Retrieved from https://www.health.govt.nz/nz-health-statistics/health-statisticsand-data-sets/obesity-statistics

Phelan, S., Burgess, D., Yeazel, M., Hellerstedt, W., Griffin, J., \& van Ryn, M. (2015). Impact of weight bias and stigma on quality of care and outcomes for patients with obesity. Obesity Reviews, 16(4), 319-326. doi:10.1 I I I/obr.12266.

Puhl, R., \& Brownell, K. (2006). Confronting and coping with weight stigma: An investigation of overweight and obese adults. Obesity: A Research Journal, 14(10), 1802-1815.

Puhl, R., Himmelstein, M., Gorin, A., \& Suh, Y. (2017). Missing the target: Including perspectives of women with overweight and obesity to inform stigma-reduction strategies. Obesity Science and Practice, 3(I), 25-35. doi:10.1002/osp4.101.

Tylka, T., Annunziato, R., Burgard, D., Daníelsdóttir, S., Shuman, E., Davis, C., \& Calogero, R. (2014). The weight-inclusive versus weight-normative approach to health: Evaluating the evidence for prioritising well-being over weight loss. Journal of Obesity, 2014(special issue), 9-26. doi:10.1155/2014/983495.

Wakefield, K., \& Feo, R. (2017). Confronting obesity, stigma and weight bias in healthcare with a person centred care approach: A case study. Australian Nursing \& Midwifery Journal, 25(I), 28-3l.

World Health Organization. (2020). Obesity and overweight. Retrieved from https://www.who.int/news-room/fact-sheets/detail/ obesity-and-overweight 